\title{
Características de deposição dos rejeitos da pilha do Monjolo com GPR
}

Rachel Jardim Martini ${ }^{*}$, Remke Leander Van Dam ${ }^{1}$, Paulo Roberto Antunes Aranha ${ }^{2}$, Hersília de Andrade e Santos ${ }^{1}$

${ }^{1}$ Programa de Pós-Graduação em Engenharia Civil do Centro Federal de Educação Tecnológica de Minas Gerais.

${ }^{2}$ Departamento de Geologia, Instituto de Geociências da Universidade Federal de Minas Gerais.

Copyright 2016, SBGf - Sociedade Brasileira de Geofísica

Este texto foi preparado para a apresentação no VII Simpósio Brasileiro de Geofísica, Ouro Preto, 25 a 27 de outubro de 2016. Seu conteúdo foi revisado pelo Comite Técnico do VII SimBGf, mas não necessariamente representa a opinião da SBGf ou de seus associados. É proibida a reprodução total ou parcial deste material para propósitos comerciais sem prévia autorização da SBGf.

\section{Resumo}

Os rejeitos, gerados pela mineração de ferro no Brasil, existem em grande quantidade e aumentam com enorme velocidade. Estes são dispostos em estruturas conhecidas como pilhas de rejeitos, que muitas vezes demandam amplas áreas dentro dos complexos minerários. A limitação dos recursos naturais e dos espaços disponíveis para armazenamento destes materiais tem levado a uma mudança de paradigma. $O$ presente estudo teve como objetivo empregar o GPR para definição da existência de padrões de sedimentação na subsuperfície da Pilha do Monjolo, em Rio Piracicaba (Minas Gerais). A partir do levantamento de perfis geofísicos, obtidos com a antena de $200 \mathrm{MHz}$, foi possível verificar diferentes texturas no subsolo. A contribuição dessas imagens para as operações da pilha é o conhecimento das linhas de deposição, que podem indicar os pontos de concentração e tendências críticas de movimentação desses rejeitos.

\section{Introdução}

O crescimento econômico do país é intimamente ligado ao desenvolvimento do setor da mineração. Percebe-se isto a partir da análise da geração de rejeitos, como exemplo da mineração de Água Limpa (Minas Gerais). Estes rejeitos são provenientes da planta de beneficiamento da usina, e são separados em três tipos: jigue, rejeito espiral e estéril.

Segundo Martini (2014), o volume de rejeito espiral possui uma maior quantidade quando comparado aos outros, em torno de $82 \%$ de toda a geração. Is so conduz a um maior interesse na compreensão do destino final e caracterização deste material.

Esta é a principal motivação para o desenvolvimento deste trabalho, que tem como objetivo analisar por meio da técnica de GPR (Ground Penetrating Radar) as características de deposição de rejeitos gerados por uma mineração de ferro. Dentre os métodos eletromagnéticos de prospecção geofísica, o GPR destaca-se por sua eficiente capacidade de obter imagens da subsuperfície e por sua fácil aplicabilidade às mais diversas situações (ANNAN, 2001). Este trabalho visa uma nova potencialidade, a utilização do GPR em ambiente de mineração.
No presente trabalho, a metodologia foi aplicada à Mina de Água Limpa, localizada no Município de Rio Piracicaba (Minas Gerais), cuja área se encontra no Quadrilátero Ferrífero. Dentro do complexo minerário, foi realizado um diagnóstico das condições atuais do empilhamento do Monjolo.

O GPR é um método que utiliza a técnica indireta para investigação das estruturas de subsuperfície. Esta tecnologia é caracterizada como método não-invasivo, o qual permite extrair informações ao longo do perfil de solo sem perfurar, sondar ou escavar.

Com este trabalho é possível avaliar a utilização desta técnica em ambientes de mineração, observando os aspectos das suas potencialidades, limitações e precisão. A possibilidade de se obter dados detalhados das camadas de rejeito de forma indireta (sem a coleta de amostras pontuais) auxilia na proposição de mecanismos de monitoramento e de controle da disposição deste material, o que aprimora as metodologias existentes e subsidia novos estudos de reutilização dos mesmos.

\section{Mineração de ferro}

A importância dos recursos minerais está vinculada ao progresso da sociedade, pois são estes recursos que contribuem para o bem estar e qualidade de vida de todas as gerações. OBrasil é um dos maiores produtores e exportadores de minérios do mundo, assim como detém um dos maiores patrimônios minerais. A mineração de ferro representa a mais importante atividade mineral do país por conta do seu efeito multiplicador e pela quantidade de recursos financeiros envolvidos (MINÉRIOS E MINERALES, 2012).

Luz e Lins (2010) descrevem que, em geral, o beneficiamento do minério de ferro compreende operações que visam modificar a granulometria, a concentração relativa das espécies minerais presentes ou a forma, sem modificar a identidade química ou física dos minerais.

De acordo com a Política Nacional de Resíduos Sólidos, lei no 12.305 , de 2 de agosto de 2010 , são considerados resíduos de mineração aqueles gerados na atividade de pesquisa, extração ou beneficiamento de minérios.

Dentre os tipos de resíduos produzidos pelas atividades mineradoras, tem-se os estéreis e os rejeitos. A norma ABNT NBR 13029:2006 conceitua estéril de mina como "todo e qualquer material não aproveitável economicamente, cuja remoção se torna neces sária para a lavra do minério". O rejeito é definido pela ABNT NBR 13028:2006 como "todo e qualquer material não aproveitável economicamente, gerado durante 0 processo de beneficiamento de minérios". 
Os rejeitos são provenientes do processo de extração dos elementos de interesse econômico da mineração, classificados como resíduos inertes. Estes são caracterizados pela presença de uma fração líquida e sólida, com concentração de $30 \%$ a $50 \%$ em peso. Caracterizam-se por serem materiais de baixa densidade, muito compres síveis e com baixa capacidade de suporte (ARAUJO, 2006; LOZANO, 2006).

O transporte dos rejeitos gerados pela atividade de mineração pode ser feito por meio de caminhões ou correias transportadoras, ou na forma de polpa (mistura de água e sólidos), transportada por meio de tubulações através da utilização de sistemas de bombeamento ou por gravidade (DUARTE, 2008).

Para a disposição final de rejeitos em pilhas, deve-se atentar para que esses depósitos não fiquem muito volumosos, pois se tornam instáveis e sujeitos a escorregamentos localizados. Além do volume oriundo do material, também devem ser consideradas as quantidades da área das jazidas e o material produzido pela decomposição das rochas e erosão do solo do entorno (SILVA, 2007).

\section{Metodologia}

O presente estudo foi realizado no complexo mineránio da Mina de Água Limpa, situado na região do Quadrilátero Ferrífero, nos municípios de Rio Piracicaba e Santa Bárbara, em Minas Gerais. A estrutura analisada foi a Pilha do Monjolo (fig. 1), na qual é feita a deposição do rejeito arenoso pelo método de lançamento hidráulico para montante.

Os parâmetros considerados para a escolha e aquisição do equipamento GPR foram definidos baseado no objetivo da pesquisa, em termos de resolução e penetração, e nas condições do meio a ser pesquisado. O GPR utilizado é da marca GSSI, modelo TerraSIR, com antena de $200 \mathrm{MHz}$. Ao GPR foi adaptado um GPS diferencial.

Foram feitos 14 perfis com a antena de $200 \mathrm{MHz}$ na estrutura da Pilha do Monjolo. A distância total percorrida foi de aproximadamente $304,7 \mathrm{~m}$.

$\mathrm{Na}$ pilha do Monjolo, a utilização do GPR exige a consideração de uma constante dielétrica diferente, por se tratar de um solo. Para aplicação nesta estrutura (fig. 2), o equipamento foi deslizado ao longo do perfil, de acordo com o método de reflexão simples. A medição do comprimento dos perfis foi feita através da survey wheel, que possui um encoder acoplado para realização desta medição.

Os perfis foram processados nos softwares Gradix Interpex, Reflexw 2D data-analysis e $\operatorname{Radan}{ }^{\circledR}$ 7. As etapas de processamento de dados GPR utilizados para os perfis da pilha do Monjolo, foram: pré-processamento; declip; análise do espectro de frequências; filtragem dewow, set time zero; window traces; remove background; filtragem; aplicação de ganho; análise de velocidades; conversão tempo/profundidade e migração.
Nos perfis obtidos foram identificadas diversas hipérboles cujas velocidades de propagação das ondas foram obtidas através dos softwares. Com este valor foi feita a correção da profundidade, através da conversão tempo/profundidade dos perfis.

Foram feitas aquisições paralelas nas bermas (superior e inferior) para identificação da variação no comportamento das linhas de deposição, levando-se em consideração uma possível diferença de compactação dos rejeitos, sendo elas os perfis 107 até 112 (fig. 3). A heterogeneidade da pilha e sua estratificação subhorizontal de camadas são causadas pelo seu método de construção. Estas camadas podem seridentificadas pelo GPR, por conta das características condutivas e resistivas dos materiais (ANTERRIEU et al., 2010).

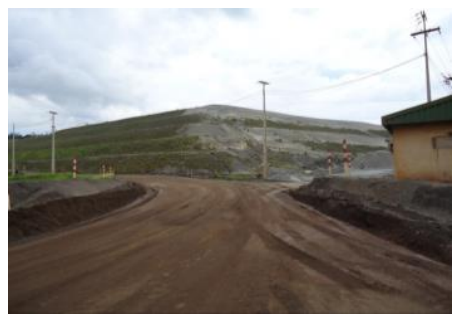

Figura 1 - Vista da pilha do Monjolo.

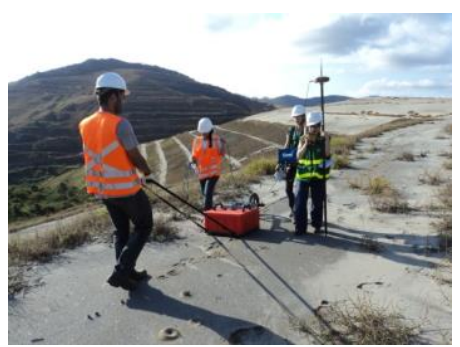

Figura 2 - Utilização do GPR na Pilha do Monjolo.

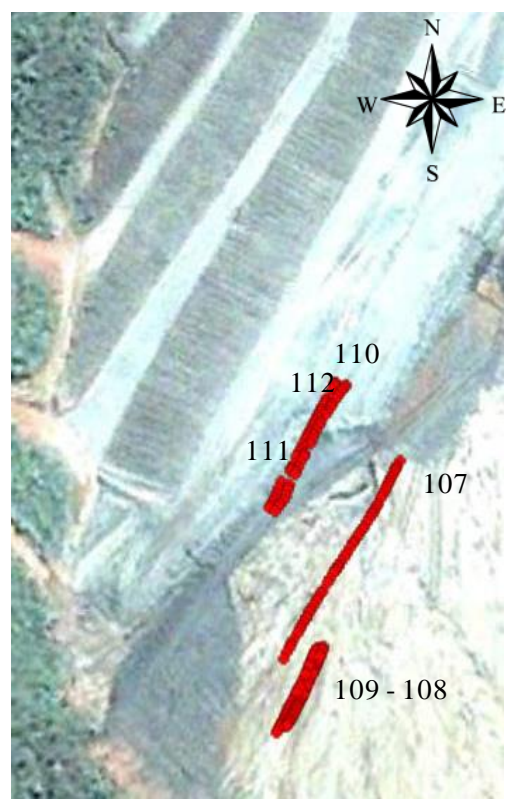

Figura 3 - Posição dos perfis. 


\section{Resultados}

Segundo Martini (2014), os rejeitos são transportados até a Pilha do Monjolo através de caminhões. Estes depositam o material na estrutura e fazem uma compactação prévia com o próprio peso dos veículos. Em todos os perfis da malha pode-se constatar um padrão irregular de deposição destacado pelo retângulo preto, assim como reflexões com amplitudes diferentes que pode representar uma atenuação do sinal por conta de presença de água ou materiais diferentes.

Os perfis 107, 108 e 109 são paralelos uns aos outros e foram obtidos nas bermas superiores (crista), determinadas, aparentemente, como não-compactadas, uma vez que foram as últimas bermas formadas por lançamento de rejeito por via de transporte por veículos. Estes perfis possuem um comprimento total aproximado de $20,50 \mathrm{~m}$ e alcançam a mesma profundidade dos demais. Nestes foram identificadas muitas hipérboles, sem um padrão de regularidade para esse comportamento. Na seção 108 (fig. 4) foi identificada uma reflexão em curva para cima (destacada em amarelo).

Os perfis 110 e 111-112 (fig. 5 e 6 ) foram amostrados paralelamente e obtidos nas bermas inferiores, determinadas, aparentemente, como compactadas. Estes possuem um comprimento total aproximado de $36 \mathrm{~m} \mathrm{e}$ alcançam a profundidade de $4,00 \mathrm{~m}$.

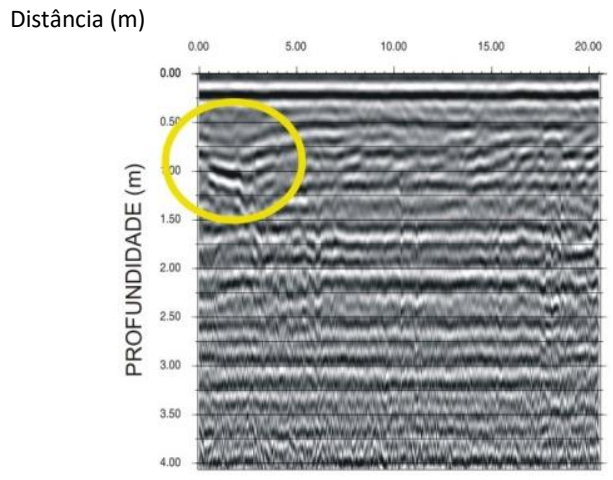

Figura 4 - Seção 108 - Direção Norte-Sul (Amplificação vertical $\approx 5$ )

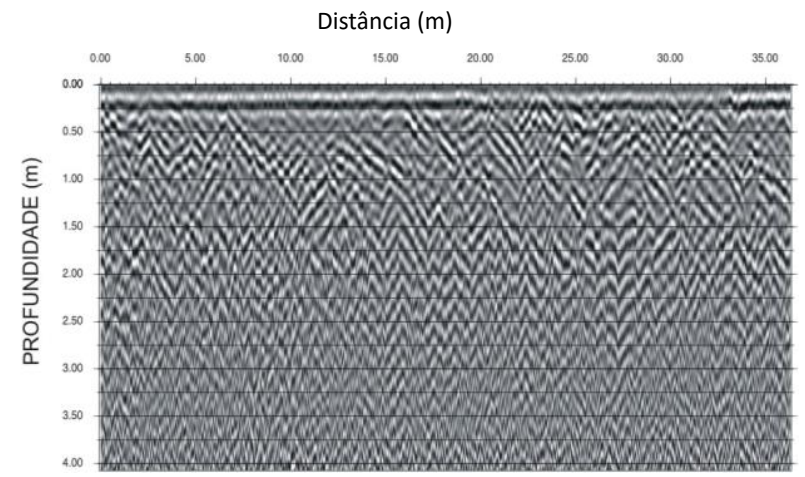

Figura 5 - Seção 110 - Direção Norte-Sul (Amplificação vertical $\approx 5$ )

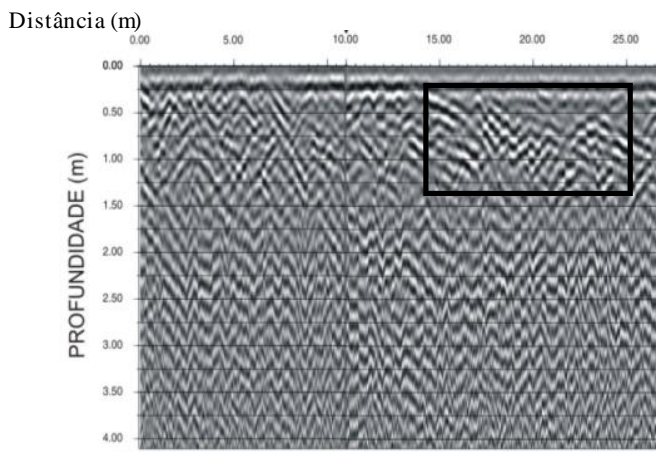

Figura 6 - Seção 111-112 - Direção Norte-Sul (Amplificação vertical $\approx 5$ )

\section{Discussão e Conclusões}

A utilização da técnica de GPR aplicada a estudos de engenharia é relativamente recente no Brasil. O presente trabalho demonstrou a possibilidade de utilização desta metodologia em monitoramento de disposição de rejeitos de minério de ferro.

Com relação aos resultados obtidos na Mina de Água Limpa, pelas análises dos perfis, pode-se destacar a concentração de água em alguns pontos devido à atenuação do sinal. Após análise dos perfis obtidos deste material, pode-se constatar que a presença de água não atenuou a propagação do sinal de GPR. Oliveira Jr. e Medeiros (2008) analisaram a influência da água, da granulometria e de minerais pesados em radargramas obtidos em rejeitos arenosos, e obtiveram uma constatação diferente. Eles alegam que o aumento do conteúdo de água em sedimentos arenosos causa aumento em sua constante dielétrica e diminuição na velocidade de propagação do sinal de GPR. Também notaram que com o aumento do teor de minerais pesados nas areias secas, o coeficiente de reflexão aumenta discretamente. Mas alguns pontos devem ser destacados: as antenas utilizadas por eles foram de 400 e $900 \mathrm{MHz}$ e o material analisado era arenoso. Para este projeto foi utilizada antena de $200 \mathrm{MHz}$ e o material é argiloso, segundo Martini (2014). Entretanto, as análises feitas por Oliveira Jr. e Medeiros (2008) foram qualitativas, pois os experimentos foram feitos em modelos reduzidos.

Segundo Parizzi et al. (2011), o radargrama permite a interpretação e visualização das estruturas de subsuperfície, e essa interpretação é feita através da análise dos padrões das ondas que geram texturas diferentes no gráfico. Essa variação se dá por conta das diferentes propriedades dielétricas da subsuperfície. Dessa forma é possível distinguir a profundidade dos meios identificados e a estimativa das espessuras. Já Cordeiro et al. (2007) afirma que a inclinação, forma e frequência das superfícies de reflexão, podem estar relacionadas à interferência sofrida pela onda emitida ao incidir sobre planos de foliação ou famílias de fraturas com direções diferentes.

Considerando estes parâmetros de interpretação, foi possível identificar a concentração de materiais em 
alguns pontos, linhas de deposição variáveis e presença de refletores.

De forma geral, as diferenças mais perceptíveis nos radargramas mostraram-se relacionadas a presença ou a ausência de refletores fortes e a espessura dos sedimentos, que variou ao longo do perfil transversal. Carvalho (2002) e Porsani et al. (2004) afirmam que a presença destes refletores observados podem ter significados geológicos desde que comparados com perfis de sondagens executados na área pesquisada.

As linhas de deposição identificadas referem-se ao histórico de deposição dos rejeitos na pilha ao longo dos anos, sendo possível identificar sua direção. Informações mais específicas como porosidade, densidade do material e espessura das camadas não foram possíveis de serem identificadas com a técnica do GPR utilizada. Entretanto, as imagens de GPR apres entaram diferenças que podem estar relacionadas à composição material (presença de hematita, argila ou areia) e ao seu teor de umidade.

A principal contribuição dessas imagens do GPR para as operações da pilha no complexo de mineração de ferro é o conhecimento da existência dessas linhas de deposição, que podem indicar os pontos de maior concentração de material, e tendências críticas de movimentação desses rejeitos.

Os resultados sugerem a utilização desta técnica em estruturas de rejeito de mineração de ferro, pois apontou que as interferências ocorridas nos radargramas por conta da composição do material (meios altamente condutivos) não foram suficientes para inviabilizar a utilização do GPR. Entretanto, a interpretação dos resultados deve ser cautelosa, podendo ser auxiliado por outras técnicas de prospecção para investigação geotécnica. Popini (2001) também afirma que possuir informações prévias sobre a geologia e as feições estruturais da área estudada pode facilitar o trabalho de interpretação dos perfis.

Portanto, a viabilidade do uso do GPR em estudos de rejeito de minério ferro, verificada neste trabalho, demonstra a possibilidade de aplicação do mesmo na área da engenharia.

Com relação à presença de materiais ferruginosos, como hematita e outros minerais de ferro, nas jazidas exploradas por mineração ou em depósitos de rejeitos destas, os estudos sobre o comportamento da reflexão da onda EM do GPR não foram, até o momento, encontrados.

A utilização do método GPR como metodologia de investigação de linha de tempo de deposição das camadas dos rejeitos na Pilha do Monjolo demonstrou-se uma técnica viável. Os níveis de interferência do rejeito de minério de ferro identificados no sinal do GPR não interferiram nos resultados alcançados.

\section{Agradecimentos}

Os autores agradecem à FAPEMIG e CAPES, pelo apoio financeiro para desenvolvimento da pesquisa. Os autores também são gratos à Lucas Ramos, Tathiana Caetano, Fernanda Cristina e Ronie Amorim pelo apoio operacional durante as pesquisas em campo.

\section{Referências}

ANNAN, A.P. Ground Penetrating Radar Workshop Notes. Sensors \& Software Inc, Canadá, 2001.

ANTERRIEU, O.; CHOUTEAU, M.; AUBERTIN, M. Geophysical characterization of the large-scale internal structure of a waste rock pile from a hard rock mine. Bull Eng. Geol. Environ, 2010. v. 69, pp. 533-548.

ARAUJO, C. B. Contribuição ao estudo do comportamento de barragens de rejeito de mineração de ferro. 2006. Dissertação (Mestrado em Engenharia) Coordenação dos Programas de Pós-Graduação de Engenharia, Universidade Federal do Rio de Janeiro, Rio de Janeiro, 2006.

ASSOCIAÇÃO BRASILEIRA DE NORMAS TÉCNICAS. NBR 13028: Mineração - Elaboração e apresentação de projeto de barragens para disposição de rejeitos, contenção de sedimentos e reservação de água. 2006.

ASSOCIAÇÃO BRASILEIRA DE NORMAS TÉCNICAS. NBR 13029: Mineração - Elaboração e apresentação de projeto de disposição de estéril em pilha. 2006.

BRASIL. Lei n 12.305, de 2 de agosto de 2010. Institui a Política Nacional de Resíduos Sólidos; altera a Lei no 9.605, de 12 de fevereiro de 1998; e dá outras providências, $2010 \mathrm{a}$.

CARVALHO, R. F. Avaliação do uso e ocupação do solo na bacia do ribeirão Serra Azul e as consequências no reservatório de abastecimento da COPASA-MG. Dissertação (Mestrado em Geografia) - Programa de Pós-Graduação do Departamento de Geografia, Universidade Federal de Minas Gerais. Minas Gerais, 2002.

CORDEIRO J. S.; FERREIRA A. F.; ARANHA P. R. A.; COSTA R. D.; FILHO J. A. S. Aplicação de GPR e cintilometria para a caracterização de descontinuidades estruturais e litológicas na porção leste da serra do Cantagalo, São Tomé das Letras (MG). Geonomos 15, v. 2, p. $21-31.2007$.

DUARTE, A. P. Classificação das barragens de contenção de rejeitos de mineração e de resíduos industriais no estado de Minas Gerais em relação ao potencial de risco. Dissertação (Mestre em Saneamento, Meio Ambiente e Recursos Hídricos) - Universidade Federal de Minas Gerais. Programa de Pós-Graduação em Saneamento, Meio Ambiente e Recursos Hídricos. Belo Horizonte, 2008.

LOZANO, F. A. E. Seleção de locais para barragens de rejeitos usando o método de análise hierárquica. 2006. Dissertação (Mestrado em Engenharia) - Escola Politécnica da Universidade de São Paulo, Universidade de São Paulo, São Paulo, 2006

LUZ, A. B. e LINS, F. A. F. Introdução ao Tratamento de Minérios. Coordenação de Processos Minerais - COPM. Rio de Janeiro, 2010.

MARTINI R. J. 2014. Aplicação do ground penetrating radar em estudos para disposição de rejeitos de minério de ferro. Dissertação (Mestrado em Engenharia) - Centro Federal de Educação Tecnológica de Minas Gerais. Belo Horizonte. 
MINÉRIOS \& MINERALES. Minérios \& Minerales apresenta $8^{a}$ edição "200 Maiores Minas Brasileiras". n. 345, p. 22-35, 2012.

OLIVEIRA JR., J. G. e MEDEIROS, W. E. Influência do conteúdo de água, granulometria e minerais pesados sobre as reflexões de GPR em corpos arenosos inconsolidados. Revista Brasileira de Geofísica, v. 26, n. 4, p. 437-449, 2008.

PARIZZI M. G.; ARANHA P. R. A.; COSTA R. D.; FILHO J. A. S.; TUPINAMBÁS M. M.; CAJAZEIRO J. M. D. Geofísica e sedimentologia aplicadas à avaliação do grau de assoreamento de trecho do Rio das Velhas em Rio Acima, Minas Gerais. Geonomos, 19, v. 2, p. 152-162. 2011.

POPINI, M. V. F. Processamento de dados de GPR utilizando métodos da sísmica de reflexão. 2001. Dissertação (Mestrado em Geofísica) - Universidade Federal da Bahia, Bahia, 2001.

PORSANI J. L.; MENDONÇA C. A.; BETTENCOURT J. S.; HIODO F. Y.; VIAN J. A. J.; SILVA J. E. Investigações GPR nos distritos mineiros de Santa Bárbara e Bom Futuro: província estanífera de Rondônia. Revista Brasileira de Geofísica, n. 22, v. 1, p. 55-66. 2004.

PORSANI J. L.; RUI Y. B.; RAMOS F. P.; YAMANOUTH G. R. B. GPR applied to mapping utilities along the route of the Line 4 (yellow) subway tunnel construction in São Paulo City, Brazil. Journal of Applied Geophysics, n. 80, p. 25-31. 2012.

SILVA, J. P. S. Impactos ambientais causados por mineração. Revista Espaço da Sophia - № 08 Novembro/2007 - Mensal - Ano I. 